\title{
Stability Limits of Capillary Bridges: How Contact Angle Hysteresis Affects Morphology Transitions of Liquid Microstructures
}

\author{
Riëlle de Ruiter, ${ }^{1}$ Ciro Semprebon, ${ }^{2}$ Mathijs van Gorcum, ${ }^{1}$ Michèl H. G. Duits, \\ Martin Brinkmann, ${ }^{2,3}$ and Frieder Mugele ${ }^{1, *}$ \\ ${ }^{1}$ Physics of Complex Fluids and MESA+ Institute for Nanotechnology, University of Twente, \\ P.O. Box 217, 7500 AE Enschede, The Netherlands \\ ${ }^{2}$ Dynamics of Complex Fluids, Max-Planck-Institute for Dynamics and Self-Organization, \\ Am Faßberg 17, 37077 Göttingen, Germany \\ ${ }^{3}$ Experimental Physics, Saarland University, 66123 Saarbrücken, Germany
}

(Received 16 October 2014; revised manuscript received 28 January 2015; published 10 June 2015)

\begin{abstract}
The equilibrium shape of a drop in contact with solid surfaces can undergo continuous or discontinuous transitions upon changes in either drop volume or surface energies. In many instances, such transitions involve the motion of the three-phase contact line and are thus sensitive to contact angle hysteresis. Using a combination of electrowetting-based experiments and numerical calculations, we demonstrate for a generic sphere-plate confinement geometry how contact angle hysteresis affects the mechanical stability of competing axisymmetric and nonaxisymmetric drop conformations and qualitatively changes the character of transitions between them.
\end{abstract}

DOI: 10.1103/PhysRevLett.114.234501

PACS numbers: 47.55.dr, 47.55.nk, 47.55.np

The physical principles governing the equilibrium shape of liquid surfaces have been known since the derivation of the basic laws of capillarity by Young, Laplace, and Dupré in the early 19th century. In the absence of gravity, liquid surfaces have a constant mean curvature and Young's angle $\theta_{Y}$ controls their intersection with solid surfaces. Despite this conceptual simplicity, capillary surfaces often display a rich and counterintuitive phenomenology with a multitude of competing mechanically stable morphologies and a pronounced history dependence that arises from the complexity of the geometries [1-4] and distributions of the local wettability [5-7] of the solid surfaces. The stability limit of such competing liquid morphologies is crucial for understanding elementary processes in capillarity such as the stability of confined liquid drops, the Cassie-Wenzel transition on superhydrophobic surfaces [8-10], the dropshedding limit on textured surfaces [11], and the mobilization of drops in complex geometries [12]. Typically, rather simplistic assumptions regarding the global geometry and wettability are made to describe such transitions between competing drop morphologies, including in particular the transition states. These simplifications frequently result in rather poor predictions and limited agreement with experimental results.

The goal of this Letter is to demonstrate the far reaching consequences of contact angle hysteresis for the stability of liquid morphologies for the specific geometry of a liquid drop confined between a flat surface and a sphere (both smooth and rigid) at a fixed distance. This geometry is representative for a wide range of trapping geometries, including pore throats in porous media. It is well known that, in the absence of contact angle hysteresis and for sufficiently small contact angles, drops placed in this geometry form cylindrically symmetric capillary bridges, whose shapes are described by Delaunay surfaces $[13,14]$. At a distance- and volume-dependent critical angle $\theta_{c}$, these morphologies become unstable and undergo a continuous transition into a symmetry-broken nonaxisymmetric state [15]. Here, we use a combination of electrowetting experiments and numerical calculations to explore the effect of contact angle hysteresis on the nature of this symmetry-breaking morphology transition. While the transition follows a pitchfork bifurcation for negligible contact angle hysteresis, we demonstrate that even minor degrees of contact angle hysteresis stabilize near-axisymmetric configurations of trapped drops for a wide range of wettabilities and geometric conditions. By stabilizing these intrinsically unstable states, contact angle hysteresis qualitatively changes the shape of the bifurcation diagram and gives rise to new discontinuous transitions between axisymmetric and nonaxisymmetric drop configurations. From a practical perspective, our results imply that the releasing and shedding of trapped drops can be substantially more difficult than expected based on common simplistic models that neglect contact angle hysteresis.

Experiments are performed by placing an aqueous drop with volume $V$ in contact with a millimeter-sized stainless steel sphere with radius $R$ and an indium tin oxide-coated glass slide that are separated by a distance $\tilde{s}$. Both substrates are covered with thin insulating layers of Parylene $\mathrm{C}$ and Teflon AF [inset of Fig. 1(a)]. The ambient medium is silicone oil, which is density matched to water to eliminate buoyancy. Electrowetting [16] is used to vary the macroscopic "Lippmann" contact angle $\theta_{L}$ of the drop on 
both solid surfaces simultaneously (see the Supplemental Material [17] for details). The contact angle hysteresis $\Delta \theta=\theta_{A}-\theta_{R}\left[\theta_{A(R)}\right.$ is the advancing(receding) contact angle] on the flat surfaces varies slightly between $<2^{\circ}$ and $\approx 5^{\circ}$ from sample to sample, which we denote as type I and type II substrates. In the experiments, the apparent contact angle $\theta$ is thus not uniquely defined because of contact angle hysteresis. Therefore, we will use the Lippmann angle $\theta_{L}$ (observed in the ideal case of vanishing hysteresis) as a control parameter. Given the small degree of hysteresis, deviations between $\theta_{L}$ and the actual contact angles $\theta$ are small. The sphere radius $R$ and the interfacial tension $\gamma$ are used to nondimensionalize all quantities (denoted by a tilde).

Initially, we deposit a drop at zero voltage $\left(\theta_{L}=\theta_{Y} \cong\right.$ $170^{\circ}$ ) next to the sphere at a radial position $\tilde{x}>1$ (Fig. 1). Upon increasing the voltage, $\theta_{L}$ decreases and the drop is gradually pulled towards the symmetry axis at $\tilde{x}=0$. Yet, even for the highest voltages applied corresponding to $\theta_{L} \approx 90^{\circ}$, the symmetry axis is not reached. While ramping down $U$ again, $\theta_{L}$ increases and the drop is pushed away from the symmetry axis, i.e., the distance $\tilde{x}$ increases as well. For type I substrates $\tilde{x}\left(\theta_{L}\right)$ largely traces back the same path as for increasing $U$ [Fig. 1(a)]. For type II surfaces, however, a pronounced hysteresis in $\tilde{x}\left(\theta_{L}\right)$ is found [Fig. 1(c)]. The nature of the hysteresis loop for the latter samples depends strongly on the minimum contact angle $\theta_{L, \text { min }}$. For rather large $\theta_{L, \min }, \tilde{x}$ decreases and increases continuously (blue symbols). For smaller $\theta_{L, \min }$, however, $\tilde{x}\left(\theta_{L}\right)$ displays a discontinuity; i.e., the drop jumps abruptly from a near-axisymmetric configuration to a pronounced off-axis position [red symbols; position 4 in Fig. 1(d)]. Video snapshots show that the drop shapes on type I substrates can be described by a section of a sphere with a $U$-dependent contact angle $\theta_{L}$ and radius $r$, whereas pronounced nonspherical surface shapes are found on type II substrates (Figs. 1(b) and 1(d), and Fig. S2 in the Supplemental Material [17]).

In mechanical equilibrium, the morphology of cylindrically symmetric capillary bridges for sufficiently small $\theta$ is described by inner nodoids or unduloids $[13,14,18]$. Such drops exert attractive forces between the confining solid surfaces [19]. At some geometry-dependent critical contact angle $\theta_{c}=\theta_{c}(\tilde{V}, \tilde{s})$ (Fig. S4 of the Supplemental Material [17]), cylindrically symmetric morphologies become unstable against nonaxisymmetric perturbations $[15,19]$. The corresponding critical drop shape is a section of a sphere [19]. For $\theta>\theta_{c}$, the drops assume a nonaxisymmetric equilibrium morphology with $\tilde{x}^{0}>0$. It is generally believed but to our knowledge not rigorously proven that the resulting equilibrium morphology is a section of a sphere that exerts zero force on the surfaces $[15,19,20]$. Assuming this conjecture to be true, a straightforward geometric analysis shows that the position of the sphere center is given by $\tilde{x}_{s}(\theta)=$ $\sqrt{\tilde{r}^{2} \sin ^{2} \theta-(2+\tilde{s})(2 \tilde{r} \cos \theta+\tilde{s})}$ [black solid lines in Figs. 1(a) and $1(\mathrm{c})]$. Here $\tilde{r}(\theta)$ is the radius of the drop,
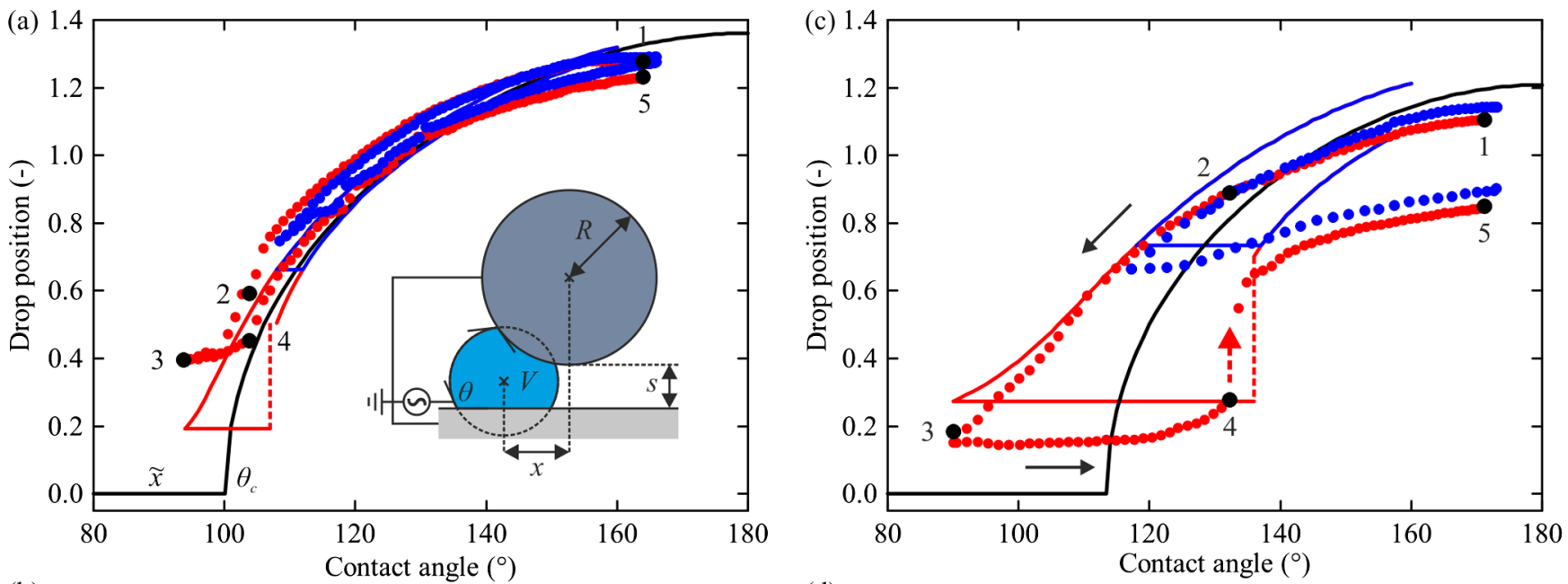

(b)

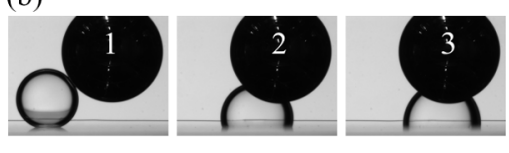

decreasing $\theta_{L}$

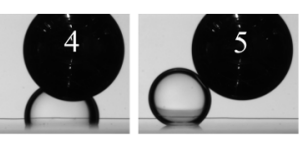

increasing $\theta_{L}$ (d)

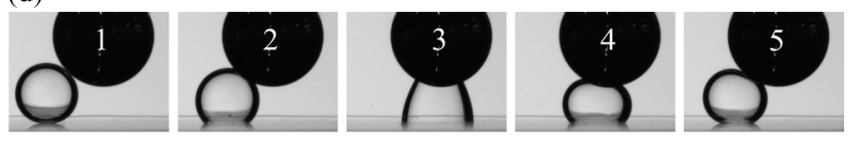

decreasing $\theta_{L}$

increasing $\theta_{L}$

FIG. 1 (color online). (a),(c) Bifurcation diagram showing drop position versus contact angle $(\tilde{V}=1$, (a) $\tilde{s}=0.5$, (c) $\tilde{s}=0.7)$. Blue and red symbols: two experimental loops of decreasing and increasing $\theta_{L}$ with different $\theta_{L, \text { min }}$. Black solid lines: geometric analysis and Surface Evolver calculations for the ideal system. Blue and red lines: model predictions for (a) $\Delta \cos \theta=0.025\left(\Delta \theta \approx 1.5^{\circ}\right)$ and (c) $\Delta \cos \theta=0.1\left(\Delta \theta \approx 6^{\circ}\right)$. Inset: drop confined in a sphere-plate geometry, with insulated electrodes. (b), (d) Snapshots at the positions indicated in (a) and (c) (see also Movies 1 and 2 and Fig. S2 in the Supplemental Material [17]). 
which varies with $\theta$ as dictated by volume conservation (Supplemental Material [17]). The spherical shape of the drops as well as the agreement between the experimental $\tilde{x}\left(\theta_{L}\right)$ and the geometric expression $\tilde{x}_{s}(\theta)$ on type I substrates [Fig. 1(a)] corroborate the above conjecture.

We support this conclusion further in numerical minimizations of the free energy $\tilde{E}=E / \gamma R^{2}=\tilde{A}_{\text {wo }}-\tilde{A}_{\text {ws }} \cos \theta$ for confined drops using the SURFACE EVOLVER [21]. Here, $\tilde{A}_{\mathrm{wo}}=A_{\mathrm{wo}} / R^{2}$ and $\tilde{A}_{\mathrm{ws}}=A_{\mathrm{ws}} / R^{2}$ are the interfacial areas of the water-oil and water-substrate interface. For each set of fixed parameters $(\tilde{V}, \tilde{s}, \theta), \tilde{E}$ is minimized under the additional global constraint of an imposed radial center of mass position $\tilde{x}_{\mathrm{cm}}=x_{\mathrm{cm}} / R$. This results in a set of drop morphologies and a corresponding energy landscape $\tilde{E}\left(\tilde{x}_{\mathrm{cm}}, \theta\right)$ [Fig. 2(a)]. For fixed $\theta<\theta_{c}$, the energy minimum is found for axisymmetric nonspherical shapes with $\tilde{x}_{\mathrm{cm}}^{0}=0$. In contrast, for fixed $\theta>\theta_{c}$ the energy minimum is found for finite values of $\tilde{x}_{\mathrm{cm}}^{0}>0$. The curve $\tilde{x}_{\mathrm{cm}}^{0}(\theta)$ that connects all the energy minima [dashed line in Fig. 2(a)] coincides with the geometric expression $\tilde{x}_{s}(\theta)$ given above. Strictly speaking, $\tilde{x}_{\mathrm{cm}}^{0}(\theta)$ and $\tilde{x}_{s}(\theta)$ are not identical. Yet, for the conditions of our experiments, the deviation between them is of the order of the line width in Fig. 1. We therefore neglect the difference in the following and

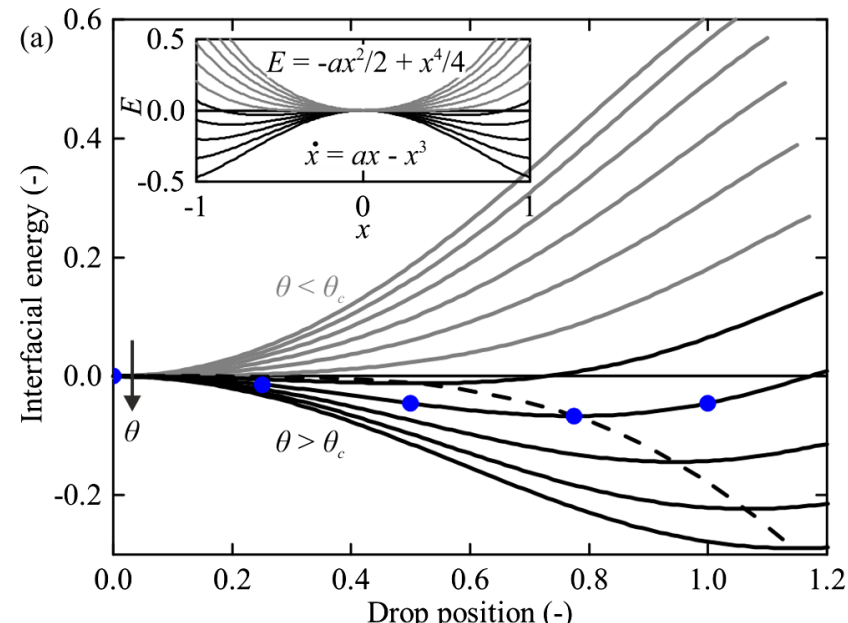

(b)

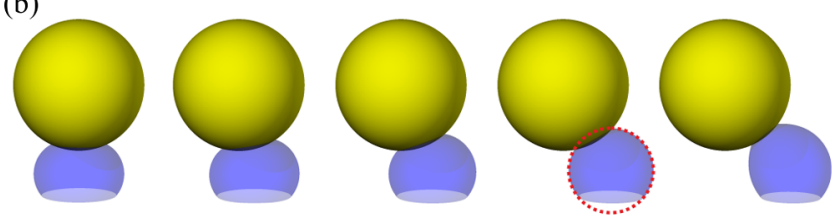

FIG. 2 (color online). (a) Energy landscape for the ideal system $\left(\tilde{V}=1, \tilde{s}=0.7, \theta=60^{\circ}, 70^{\circ}, \ldots, 160^{\circ}\right.$ ) (see also Fig. S5 and Movie S3 in the Supplemental Material [17]). Offsets are subtracted such that all axisymmetric shapes have zero interfacial energy. Dashed line: minimum energy configurations. Inset: potential for the normal form of a supercritical pitchfork bifurcation. (b) Numerically computed drop shapes for $\theta=130^{\circ}$, corresponding to the blue symbols in (a). parametrize both equilibrium configurations as $\tilde{x}^{0}(\theta)$, omitting the subscripts.

Moreover, the equilibrium drop shapes for $\theta>\theta_{c}$ are sections of spheres, as conjectured. In contrast, drops with imposed $\tilde{x} \neq \tilde{x}^{0}(\theta)$ assume nonspherical morphologies [Fig. 2(b)]. Such drops experience finite capillary forces $\tilde{F}_{\gamma}=-d \tilde{E} / d \tilde{x}$ in the radial direction pushing them towards the equilibrium position $\tilde{x}^{0}(\theta)$. While neither experiments nor numerical calculations can provide proof in a mathematical sense, the consistency of these results strongly supports the conjecture that the equilibrium shape of confined drops in a sphere-plate geometry for $\theta>\theta_{c}$ is indeed a section of a sphere, with a radial position that simply follows from the parameters $\tilde{V}, \tilde{s}$, and $\theta$.

The numerical analysis also demonstrates the absence of energy barriers, indicating that the transition between axisymmetric and nonaxisymmetric morphologies at $\theta=$ $\theta_{c}$ should be continuous and fully reversible, in contrast to various other morphology transitions $[3,22]$. The energy landscape can be approximated by a combination of a fourth order term in $\tilde{x}$ with a positive coefficient and a quadratic term with a coefficient $a$ that changes sign at $\theta=\theta_{c}$ [inset of Fig. 2(a)]. Such a structure is characteristic of a supercritical pitchfork bifurcation [23] with a manifold of symmetry-broken stable states (with arbitrary azimuthal angle) with $\tilde{x}^{0}>0$ for $\theta>\theta_{c}$ and a single symmetric solution with $\tilde{x}^{0}=0$ for $\theta<\theta_{c}$. The latter still exists for $\theta>\theta_{c}$, but is unstable against infinitesimal perturbations.

The nonspherical drops on type II substrates assume configurations with $\tilde{x} \neq \tilde{x}^{0}$ [Fig. 1(c)]. Hence, they experience radial capillary forces $\tilde{F}_{\gamma}(\tilde{x})$. Because the drops are mechanically stable at these nonequilibrium positions, $\tilde{F}_{\gamma}$ must be balanced by other forces, namely by pinning forces due to the small but finite contact angle hysteresis on these type II substrates. Ignoring possible local relaxation mechanisms [24], we approximate the maximum pinning force as $\tilde{F}_{p}=F_{p} / \gamma R=\tilde{W} \Delta \cos \theta$. Here, $\Delta \cos \theta=\cos \theta_{R}-$ $\cos \theta_{A}$ and $\tilde{W}=W / R$ is the total width of the interfacial areas of the drop on both substrates, i.e., the plate and the sphere [25-27]. The curves $\tilde{W}=\tilde{W}(\theta, \tilde{x})$ are extracted from the numerically obtained drop shapes described above (Supplemental Material [17]). We define normalized radial forces as $\tilde{F}_{r}(\theta, \tilde{x})=\tilde{F}_{\gamma} / \tilde{F}_{p}$ and plot them in Fig. 3(a) for the specific conditions $(\tilde{V}, \tilde{s}, \Delta \theta)$ of Fig. 1(c). Any drop configuration in Fig. 3(a) with $\left|\tilde{F}_{r}(\theta, \tilde{x})\right|<1$ corresponds to a mechanically stable configuration because $\tilde{F}_{p}$ exceeds $\tilde{F}_{\gamma}$ under these conditions. Drop configurations with $\tilde{F}_{r}(\theta, \tilde{x})>1$ are unstable and experience a net force pointing radially outward. Only under this condition are capillary forces strong enough to push the drop outward against the pinning forces. Such states exist only if $\theta$ exceeds a critical value $\theta_{p}$, see the star in Fig. 3(a) that 

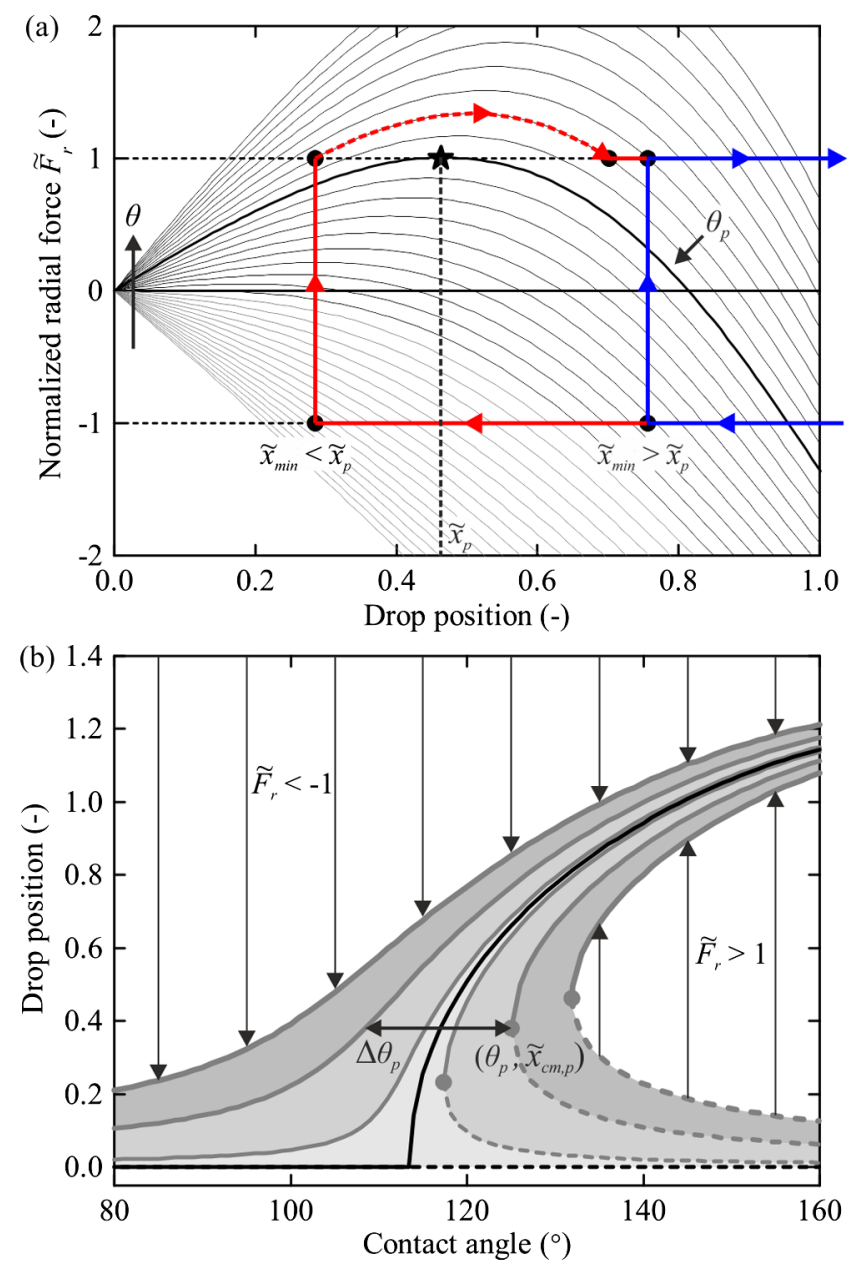

FIG. 3 (color online). (a) Ratio of the capillary force and the maximum pinning force versus drop position $(\tilde{V}=1, \tilde{s}=0.7$, $\left.\theta=80^{\circ}, 82^{\circ}, \ldots, 150^{\circ}\right)$. Blue and red arrows: two paths for decreasing and increasing $\theta$ with different $\tilde{x}_{\min }$. (b) Corresponding bifurcation diagram for $\Delta \cos \theta=0.01,0.05$, and 0.1 (increasing line thickness). Solid and dashed lines: stable and unstable limits. Black line: pitchfork bifurcation in the absence of contact angle hysteresis.

indicates the critical state with $\tilde{F}_{r}\left(\theta_{p}, \tilde{x}_{p}\right)=1$. For $\theta<\theta_{p}$, $\tilde{F}_{r}(\theta, \tilde{x})<1$ for all drop positions, implying that capillary forces are never strong enough to push drops away from the symmetry axis, independent of $\tilde{x}$. Similarly, configurations with $\tilde{F}_{r}(\theta, \tilde{x})<-1$ imply inward-oriented net forces. Such states exist for all $\theta$ provided that $\tilde{x}$ is large enough. Drops forced into states with $\left|\tilde{F}_{r}(\theta, \tilde{x})\right|>1$ relax until they reach a position $\tilde{x}^{h}(\theta)$ with $\left|\tilde{F}_{r}\left(\theta, \tilde{x}^{h}\right)\right|=1$. Provided that inertial effects are negligible, we expect that the drop follows the curve $\tilde{F}_{r}(\theta, \tilde{x})$ during relaxation.

In our experiments, we impose a quasistatic variation of $\theta_{L}$. Translating the experimental protocol to Fig. 3(a), the above considerations imply that the drop moves radially inward along $\tilde{F}_{r}\left(\theta, \tilde{x}^{h}\right)=1$ upon decreasing $\theta_{L}$. As $\theta_{L}$ is reversed at $\theta_{L, \min }$, the drop remains at the fixed location $\tilde{x}_{\min }$ until the state $\tilde{F}_{r}\left(\theta, \tilde{x}_{\min }\right)=1$ is reached. If $\tilde{x}_{\min }>\tilde{x}_{p}$ that state is mechanically stable thanks to the negative slope of $\tilde{F}_{r}$ at that location. Hence, the drop continuously moves radially outward along $\tilde{F}_{r}\left(\theta, \tilde{x}^{h}\right)=1$ as $\theta_{L}$ is further increased [blue trajectory in Fig. 3(a)]. In contrast, if $\tilde{x}_{\min }<$ $\tilde{x}_{p}$ the state with $\tilde{F}_{r}\left(\theta, \tilde{x}_{\min }\right)=1$ is reached in a mechanically unstable configuration with a positive slope of $\tilde{F}_{r}$. Inertial effects pending, the drop abruptly moves along the dashed red line in Fig. 3(a) until it reaches a mechanically stable state with $\tilde{F}_{r}\left(\theta, \tilde{x}^{h}\right)=1$. The appearance of discontinuities in the drop position thus arises from the mechanical instability of states with $\tilde{F}_{r}\left(\theta, \tilde{x}^{h}\right)=1$ for $\theta>\theta_{p}$ and $\tilde{x}_{\min }<\tilde{x}_{p}$.

Mapping the stable and unstable states with $\left|\tilde{F}_{r}(\theta, \tilde{x})\right|<$ 1 and $\left|\tilde{F}_{r}(\theta, \tilde{x})\right|>1$ back to the experimentally accessible parameter space $\left(\theta_{L}, \tilde{x}\right)$, we find that the ideal curve $\tilde{x}^{0}(\theta)$ of true equilibrium drop configurations [black solid line in Fig. 3(b)] is surrounded by a region of mechanically stable drop configurations held in place by pinning forces (gray area). The boundaries of this region (solid gray lines) correspond to states with $\left|\tilde{F}_{r}(\theta, \tilde{x})\right|=1$. The width of the pinning region increases with increasing $\Delta \theta$ (Fig. 3(b), and Fig. S9 of the Supplemental Material [17]). Vice versa, for $\Delta \theta \rightarrow 0$, it collapses to zero and $\tilde{x}^{0}(\theta)$ is recovered as a unique stable solution, as expected. Drops forced into unstable states (white areas) are attracted towards the boundary of the pinning-stabilized region, as indicated by the arrows in Fig. 3(b). Interestingly, contact angle hysteresis not only broadens the stable solutions of the pitchfork bifurcation into zones of finite width, it also introduces a new mechanically stable region around the unstable solution branch at $\tilde{x}=0$ for $\theta>\theta_{p}$. The finite width of that zone for arbitrarily small $\Delta \cos \theta$ implies that drops placed exactly on the symmetry axis remain stable even for very large $\theta_{L}$.

To quantitatively compare the model predictions to the experiments, we map the red and blue drop trajectories from Fig. 3(a) to the $\left(\theta_{L}, \tilde{x}\right)$ space [solid red and blue lines in Figs. 1(a) and 1(c)] using contact angle hysteresis as a fit parameter. Optimum values $\Delta \cos \theta=0.025$ and 0.1 corresponding to $\Delta \theta=1.5^{\circ}$ and $6^{\circ}$ (for $50^{\circ}<\theta_{L}<130^{\circ}$ ) for type I and type II substrates were found, consistent with the contact hysteresis on the flat surfaces. Without any additional fit parameter beyond $\Delta \theta$, all salient features of the measurements are reproduced, including in particular the appearance of the pronounced discontinuity in $\tilde{x}$ for sufficiently small $\theta_{L, \min }$ on the type II substrates. On type I surfaces, this discontinuity is still present in the model, yet it is not perceived in the experiments. Similarly, the absolute value of $\tilde{x}_{\min }$, the expected constancy of $\tilde{x}_{\min }$ upon reversing $\theta$ [between points 3 and 4 in Figs. 1(a) and 1(c)], as well as the opening of the hysteresis loop for a full cycle of increasing and decreasing $\theta_{L}$ (point 1 versus point 5) are 
not perfectly reproduced. We attribute these deviations to a combination of several effects. First, a detailed analysis of the video data suggests that drop motion is sometimes facilitated by local rearrangements of the contact line position before the entire drop starts to move. Such processes promote drop mobilization and effectively reduce the net pinning force [24,28]. Moreover, electrowettingspecific phenomena may lead to a slight decrease of $\theta_{Y}$ and an associated increase of $\tilde{F}_{p}$, due to the squeeze out of the residual oil between the drop and substrate during the excursion to the highest voltage [29].

Physically, the stabilization of ideally unstable drop morphologies with $\tilde{x} \ll 1$ is caused by the vanishing capillary forces $\tilde{F}_{\gamma}$ for $\tilde{x} \rightarrow 0$. Because driving forces always vanish close to any local maximum of an energy landscape in an arbitrary configurational space, the same type of stabilization is expected upon introducing Coulomb-like friction forces into arbitrary systems that display a supercritical pitchfork bifurcation in the absence of friction. Indeed dynamical mechanical systems displaying friction-induced stabilization have occasionally been studied [30]. In the context of microfluidics, any morphology transition that involves moving contact lines and energy landscapes of the generic type considered here will be affected. For the specific case of confined drops, the hysteresis-induced stabilization should have important practical consequences, e.g., for the mechanical stability of wet granular media [31,32] and for drop-based actuators [33]. For applications such as two phase flows in porous media, contact angle hysteresis is expected to stabilize trapped drops at pore throats and to hinder their removal.

In conclusion, the hysteresis-induced stabilization of otherwise unstable liquid morphologies is very general. It is expected to apply in many situations, including the shedding and release of drops in oil recovery and other practically relevant circumstances.

We acknowledge financial support by the Exploratory Research Program (ExploRe) of BP plc, and by the Nederlandse Organisatie voor Wetenschappelijk Onderzoek (NWO, Netherlands Organisation for Scientific Research) and the Stichting voor de Technische Wetenschappen (STW, Foundation for Technical Science) within the Vici program. We thank Daniel Wijnperle for technical support. We would like to thank T. I. Vogel and J. C. Bird for helpful comments.

R. R. and C.S. contributed equally to this work.

*f.mugele@utwente.nl

[1] R. Seemann, M. Brinkmann, E. J. Kramer, F. F. Lange, and R. Lipowsky, Proc. Natl. Acad. Sci. U.S.A. 102, 1848 (2005).

[2] E. A. Theisen, M. J. Vogel, C. A. Lopez, A. H. Hirsa, and P. H. Steen, J. Fluid Mech. 580, 495 (2007).
[3] H. B. Eral, J. de Ruiter, R. de Ruiter, J. M. Oh, C. Semprebon, M. Brinkmann, and F. Mugele, Soft Matter 7, 5138 (2011).

[4] R. de Ruiter, J. de Ruiter, H. B. Eral, C. Semprebon, M. Brinkmann, and F. Mugele, Langmuir 28, 13300 (2012).

[5] P. Lenz and R. Lipowsky, Phys. Rev. Lett. 80, 1920 (1998).

[6] H. Gau, S. Herminghaus, P. Lenz, and R. Lipowsky, Science 283, 46 (1999).

[7] C. Schafle, M. Brinkmann, C. Bechinger, P. Leiderer, and R. Lipowsky, Langmuir 26, 11878 (2010).

[8] M. Reyssat, J. M. Yeomans, and D. Quere, Europhys. Lett. 81, 26006 (2008).

[9] H. M. Kwon, A. T. Paxson, K. K. Varanasi, and N. A. Patankar, Phys. Rev. Lett. 106, 036102 (2011).

[10] P. Papadopoulos, L. Mammen, X. Deng, D. Vollmer, and H. J. Butt, Proc. Natl. Acad. Sci. U.S.A. 110, 3254 (2013).

[11] J. B. Boreyko and C.-H. Chen, Phys. Rev. Lett. 103, 184501 (2009).

[12] Y. Zheng, H. Bai, Z. Huang, X. Tian, F.-Q. Nie, Y. Zhao, J. Zhai, and L. Jiang, Nature (London) 463, 640 (2010).

[13] C. Delaunay, J. Math. Pures Appl. 6, 309 (1841).

[14] J. Plateau, The Annual Report of the Smithsonian Institution (1864), p. 338.

[15] T. I. Vogel, Pac. J. Math. 224, 367 (2006).

[16] F. Mugele and J. C. Baret, J. Phys. Condens. Matter 17, R705 (2005).

[17] See Supplemental Material at http://link.aps.org/ supplemental/10.1103/PhysRevLett.114.234501 for the geometric analysis to obtain the bifurcation diagram, and additional figures and movies.

[18] F. M. Orr, L. E. Scriven, and A. P. Rivas, J. Fluid Mech. 67, 723 (1975).

[19] R. K. Niven, J. Pet. Sci. Eng. 52, 1 (2006).

[20] T. I. Vogel (private communication).

[21] K. A. Brakke, Exp. Math. 1, 141 (1992).

[22] G. McHale and M. I. Newton, Colloids Surf. A 206, 79 (2002).

[23] S. H. Strogatz, Nonlinear Dynamics and Chaos: With Applications to Physics, Biology, Chemistry, and Engineering (Westview Press, Boulder, 1994).

[24] C. Semprebon and M. Brinkmann, Soft Matter 10, 3325 (2014).

[25] C. G. Furmidge, J. Colloid Sci. 17, 309 (1962).

[26] E. B. Dussan and R. T. P. Chow, J. Fluid Mech. 137, 1 (1983).

[27] D. 't Mannetje,A. Banpurkar, H. Koppelman, M. H. G. Duits, D. van den Ende, and F. Mugele, Langmuir 29, 9944 (2013).

[28] M. Musterd, V. van Steijn, C. R. Kleijn, and M. T. Kreutzer, Phys. Rev. Lett. 113, 066104 (2014).

[29] A. Staicu and F. Mugele, Phys. Rev. Lett. 97, 167801 (2006).

[30] H. Hetzler, in 11th International Conference on Vibration Problems, edited by Z. Dimitrovová et al. (ICOVP, Portugal, 2013).

[31] M. Scheel, R. Seemann, M. Brinkmann, M. Di Michiel, A. Sheppard, B. Breidenbach, and S. Herminghaus, Nat. Mater. 7, 189 (2008).

[32] E. Koos and N. Willenbacher, Science 331, 897 (2011).

[33] M. J. Vogel and P. H. Steen, Proc. Natl. Acad. Sci. U.S.A. 107, 3377 (2010). 